\title{
REPORT
}

\section{Comissioner of Agriculture} ANU

\section{IMNIGRATION}

$$
\text { UF THE }
$$

STATE OF NEW HAMPSHIRE,

$$
\text { Tก THE }
$$

GUVERNOR IND COLNCH.

$$
A \cup G \cup S T, 1890
$$

MANCHESTER, N. H. :

JUIIN II, CIIRKF, PUILIC PRINTER. 1890 . 


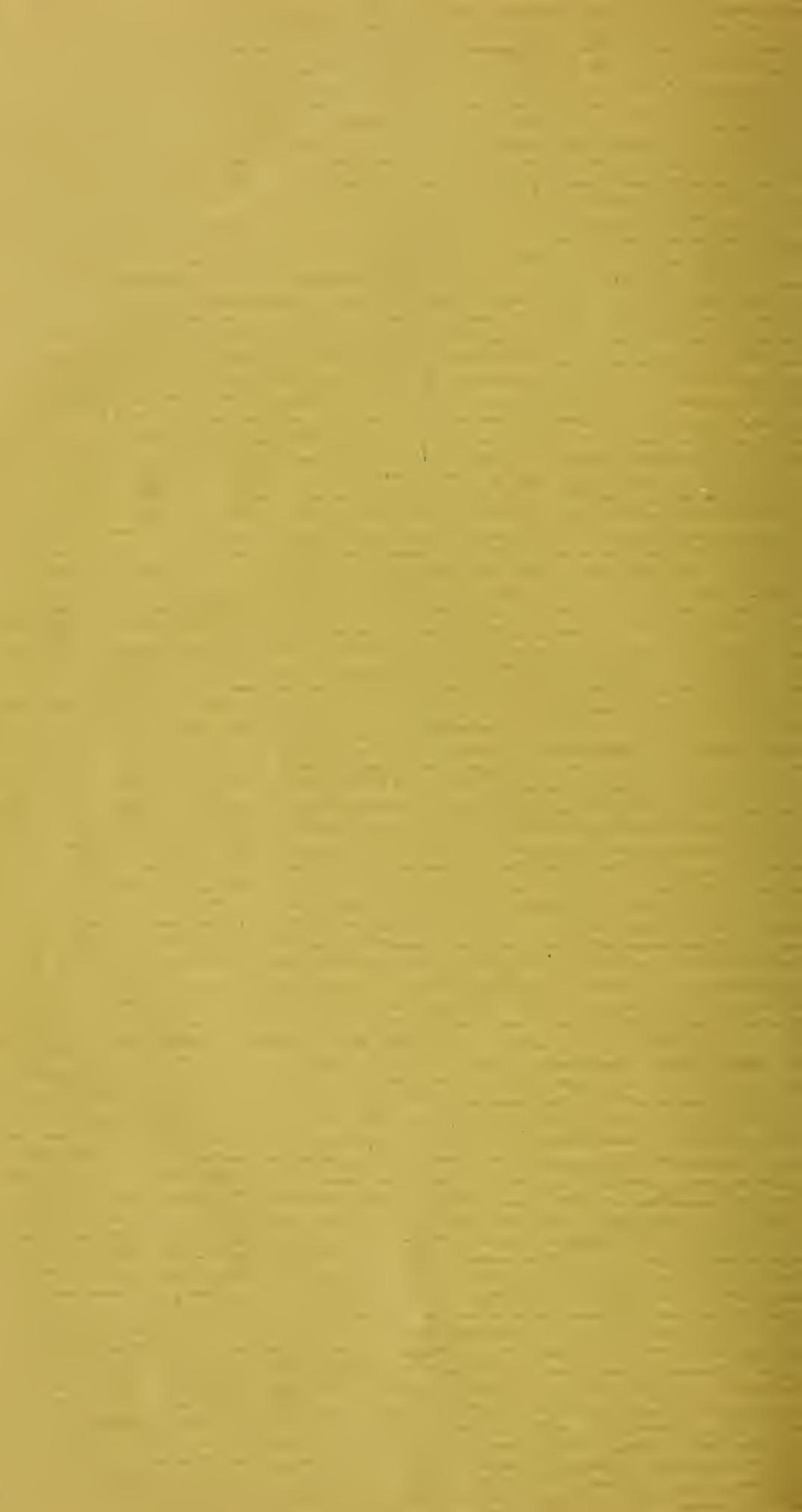






\title{
REPORT
}

OF THE

\section{Commissioner of Agriculture \\ AND}

\section{IM MIGRATION}

OF THE

\begin{abstract}
STATE OF NEW HAMPSHIRE,
\end{abstract}
TO THE

GOVERNOR AND COUNCIL.

$A \cup G \cup S T, 1890$.

MANCHESTER, N. H.:

JOHN B. CLARKE, PUBLIC PRINTER.

I $S 90$. 
सहों

. $A 32$

f.

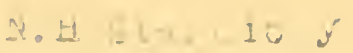

$20 \ldots 3$ 


\section{R E P O R T}

OF THE

\section{COMMISSIONER OF AGRICULTURE ANI IMMIGRATION.}

Concord, August i $2, \mathrm{I} 890$.

\section{To His Excellency Gov. D. H. Goodell, and the Honorable Council:}

A commission was received August 7, I 889, from your honorable body, authorizing us to carry into effect the law passed at the last session of the Legislature entitled "an act for the development of the agricultural resources of the State through immigrations and other means." Although this measure had been passed with great unanimity by both branches of the Legislature, and had been given universal support by the press and citizens generally, it was not an easy matter to decide npon the most judicious course to be taken under its provisions. There was no precedent to follow, and whaterer action might be taken could only be regarded by your commissioner as somewhat experimental, each step being attended with some hesitancy and doubt. During the past few years frequent reference has been made to the large number of abandoned farms in the State, many possessing advantages superior to occupied farms yielding to their occupants and owners profitable and satisfactory returns, causing a universal feeling of regret by people of every class and profession. In fact it was largely due to the sentiment prevailing in regard to the abandoned farms that the law was enacted, 
and to secure their re-occupancy have our efforts thus far been mainly directed. We herewith submit for your consideration the following brief report.

While we are able to report in detail the various means we have employed, it will be impossible to state with any degree of exactness the effect upon the prosperity of the State produced by the enactment of the law. Not only has the subject been fully and favorably discussed by the press of the State, but the same powerful agency outside New Hampshire has been actively interested in the work, and newspapers having a national reputation have devoted columns of valuable space to animated descriptions of the attractions of the Granite State and the advantages of securing a home within her borders. Organizations, both agricultural, financial, and social have discussed the subject at their gatherings, thus giving it the widest possible publicity. Even the reference made by a representative from a Western State in debate in the halls of Congress indicates the wide-spread notice given to our efforts, and doubtless aided in directing attention to these lands. In addition to all this, thinking, acting, working farmers from one end of the State to the other have been pondering over the condition of affairs in our rural towns thus brought to public attention, and in their minds has developed a desire to know the necessary course to be pursued to prevent further depletion of the agricultural population. It may be safely assested that the realizing sense of the situation has already led to the beginning of a more thoughtful and intelligent system of farming and a higher appreciation of the opportunities that surround us. These are results that cannot be measured or even indicated by statistics at the present time.

As before stated, it was with more or less hesitancy at first that we entertained the proposition to advertise to the world that within the borders of our beautiful State were hundreds of abandoned farms, but recognizing the business principle that no one comes to purchase until he knows what there is for sale, we entered upon the work, and at no time have we had any reason to regret the course taken. August 24 , I $8 S_{9}$, the following circular letter was addressed to the selectmen of each town in the State, accompanied with a blank for their report: 
STATE OF NEW HAMPSHIRE.

OFFICE OF

Commissioner of Agriculture and Immigration,

CoNCord, August 24, 1 SS9.

To the Sei.ectaien of New Hampshrire:

Gentlemen, - Having been appointed by His Excellency Governor Goodell to carry into effect the provisions of a law enacted at the recent session of the Legislature for the "development of the agricultural resources of the state through immigration and other means," we desire the co-operation and assistance of the citizens of the State, and especially of the town officers to whom this circular letter is addressed. The prime object of the measure is the repopulation of the abandoned farms, at the same time expecting to stimulate and encomrage the business of husbandry in all sections of our state.

The best course of procedure is not yet fully determined, but it is plainly evident that we must first secure a list of the fams in the interest of which we are directly working. The selectmen of the towns are therefore requested to forward to this office, on the blank inclosed, the name and address of the owner of each abandoned farm in their respective towns, on which are buildings that would afford a comfortable home for the occupant. Also please report on the same blank, as indicated, the estimated acreage of all abandoned farms on which there are no buildings, or none that you consider can be made suitable for occupancy at little expense, designating the acreage in woodland, pasturage, and tillable or meadow land under the head of mowing land. This class would not include any farm that had been abandoned and is now being operated by the occupant of an adjoining farm, while those in the former class are to be reported whatever their present management may be. Also please report the name and address of the owner of any farm occupied and for sale, as means may be taken to give some assistance to this class, under certain requirements to be hereafter announced.

We are aware that the law does not make it obligatory on the part of the selectmen to furnish this information, yet we feel sure that your interest in the work and in the development of your town and the State will prompt you to send the information which is more readily at your command than at that of any other citizen of your town. $\Lambda \mathrm{fter}$ your report is received, carefully prepared blanks will he sent to the owners of the farms asking for the necessary facts in making the advantages of the same known. The receipt of these reports will enable us to learn the locality where there are the greatest number of abandoned farms, and those sections offering the greatest inducements to settlers will he visited by the commissioner for the purpose of oltaining additional facts necessary for the encouragement of settlements. You will occasionally be informed in regard to the work of this department, which, we trust, with the assistance and co-operation of the citizens of the 
State, may be successful in turning the attention of people toward our rural towns, and in due course of time result in the repopulation and cultivation of many abandoned farms. In this work we ask your cordial co-operation.

\section{N. J. BACHELDER,}

Commissioner.

It will be remembered that the law did not require this investigation on the part of the selectmen, and it is only just that we here acknowledge their uniform courtesy in replying. Reports were received from 2 I 2 towns, of which $5^{8}$ reported no abandoned farms of the class first mentioned, and 154 towns reported r,342 abandoned farms with tenantable buildings.

The number of acres in farms abandoned on which were no buildings and not made an adjunct of some adjoining farm was small, and the prospect of accomplishing anything in the re-occupancy of these lands was not considered sufficiently encouraging to warrant any special effort at the present time.

Blanks were immediately sent to the address of the owner of each abandoned farm, requiring a reply to stated questions in regard to the farm, and the price for which it would be sold. The accuracy of the statements made and obligation to sell for the price named were to be accompanied in every instance by the owner's affidavit before a justice of the peace. Various sections of the State were visited, especially those where the greatest number of abandoned farms were reported, in order to become more familiar with their condition and surroundings. From the description of farms received and from observations thus made a pamphlet of 103 pages was prepared, containing a map of the State and statements in regard to the financial, educational, and social advantages of New Hampshire. The adaptability of the abandoned farms to various branches of agriculture was discussed and special prominence given to their advantages for summer homes. A list of I,300 summer hotels and boarding-houses in the State was included in the pamphlet, and statistics given in regard to this rapidly increasing New Hampshire industry. A complete list of creameries with their location was also included, and numerous illustrations of typical New Hampshire scenery. An edition of 8,000 copies was published and 6,000 copies have been distributed, having been sent to all parts of the United 
States, and even to Canada, England, and Sweden. Over two thousand letters of inquiry have been received, and the questions therein asked in regard to the abandoned farms of the State have been promptly and as fully answered as the information at our command would allow. Although a matter of not much importance, it may be one of some interest to state where these letters came from. The number of inquiries received from the various localities is as follows:

\begin{tabular}{|c|c|c|c|c|c|c|c|c|c|c|c|}
\hline \multirow{2}{*}{\multicolumn{2}{|c|}{$\begin{array}{l}\text { California . } \\
\text { Connecticut }\end{array}$}} & - & $\cdot$ & • & . 6 & New Yor & & . & & & . 244 \\
\hline & & . & $\bullet$ & . & - 39 & North $C_{a}$ & rolina & . & & & . I I \\
\hline Florida & . & - & & & . 3 & Ohio . & . & . & & & 28 \\
\hline Georgia & . & . & & & I & Oregon & . & . & & & . \\
\hline Illinois & . & . & & & . 48 & Pennsylv & ania & . & & & 54 \\
\hline Indiana & . & - & 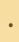 & . & . $\quad 12$ & Rhode Is & land & . & & & I 7 \\
\hline Iowa & . & . & & & . 27 & South $\mathrm{D}$ & kota & . & & & . \\
\hline Kansas & . & . & & & 27 & South $\mathrm{Ca}$ & rolina & . & & & . \\
\hline Kentucky & . & . & . & • & I & Tennesse & & . & & & . \\
\hline Maine & . & . & & & 44 & Texas & • & - & & & . \\
\hline Maryland & . & . & & - & . 34 & Vermont & . & . & & & 90 \\
\hline Massachus & etts & . & • & • & . 975 & Virginia & . & . & & & . \\
\hline Michigan & . & . & & & - 36 & West Vir & ginia & . & & & . \\
\hline Minnesota & . & . & - & . & . 15 & Wisconsi & & . & & & I 4 \\
\hline Missouri & . & - & . & - & . 5 & IVashingt & & . & & & . \\
\hline Nebraska & . & . & . & - & - 10 & Canada & . & $\cdot$ & & & . 30 \\
\hline North Dal & cota & . & • & . & . $s$ & London & Engla & nd) & & & . 2 \\
\hline New Ham & pshire & & 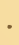 & 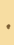 & . 422 & & & & & & \\
\hline New Jerse & & - & 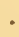 & & - 30 & & & & & & 2,257 \\
\hline
\end{tabular}

Following will be found the number of abandoned farms in each town reported by the selectmen August 24, r889, the number sold and occupied since the above date, also the number which have become occupied by a tenant or the owner in addition to those sold. The fourth column represents the total number of farms vacant at the passage of this law and now occupied by the purchaser, former owner, or tenant. 


\begin{tabular}{|c|c|c|c|c|}
\hline Towns. & 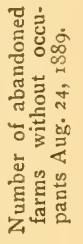 & 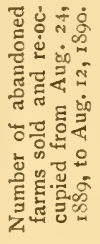 & 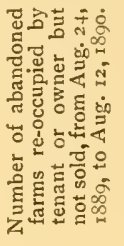 & 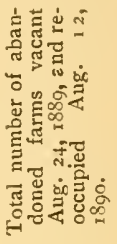 \\
\hline 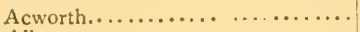 & 22 & 2 & 3 & 5 \\
\hline Albany....$\ldots \ldots \ldots \ldots \ldots \ldots \ldots$ & 6 & - & $\therefore$ & . \\
\hline Alexandria...$\ldots \ldots \ldots \ldots \ldots \ldots$ & 13 & 1 & .. & I \\
\hline 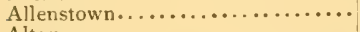 & 7 & . & 3 & 3 \\
\hline Alton $\ldots \ldots \ldots \ldots \ldots \ldots \ldots \ldots \ldots$ & 12 & I & .. & I \\
\hline Alstead...$\ldots \ldots \ldots \ldots \ldots$. & 18 & $\mathbf{I}$ & 4 & 5 \\
\hline Amhersi ...................... & 4 & $\cdots$ & $\cdots$ & -. \\
\hline Andover...$\ldots \ldots \ldots \ldots \ldots \ldots \ldots$ & 2 & . & -. & .. \\
\hline Antrim............................ & 7 & . & $\cdots$ & . \\
\hline 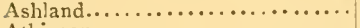 & 7 & . & 3 & 3 \\
\hline Atkinson...................... & 4 & . & .. & . \\
\hline Auburn ....................... & 3 & I & .. & 1 \\
\hline Barrington...$\ldots \ldots \ldots \ldots \ldots \ldots$ & 10 & 2 & .. & 2 \\
\hline Bartett ....................... & 5 & 2 & .. & 2 \\
\hline Bath........................ & IS & $\mathbf{I}$ & 7 & 8 \\
\hline Bedford $\ldots \ldots \ldots \ldots \ldots \ldots \ldots \ldots \ldots$ & 4 & .. & i & I \\
\hline Belmont...................... & 3 & I & $\cdots$ & 1 \\
\hline Benton...................... & 16 & $\cdots$ & 2 & 2 \\
\hline Bennington.... . ............... & 2 & .. & 2 & 2 \\
\hline 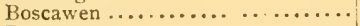 & 5 & I & $\mathbf{1}$ & 2 \\
\hline Bow $\quad, \quad \ldots \ldots \ldots \ldots \ldots \ldots \ldots \ldots$ & 6 & .. & .. & .. \\
\hline Bradford $\ldots \ldots \ldots \ldots \ldots \ldots \ldots \ldots$ & 8 & I & $\mathbf{I}$ & 2 \\
\hline Bridgewater...... . $\ldots \ldots \ldots \ldots$ & 7 & 2 & $\mathbf{I}$ & 3 \\
\hline Bristol.. $\quad \ldots \ldots \ldots \ldots \ldots \ldots$ & I & .. & .. & .. \\
\hline Brookline...$\ldots \ldots \ldots \ldots$ & II & 4 & 3 & 7 \\
\hline Campton ...................... & ro & $\because$ & I & $\mathbf{I}$ \\
\hline 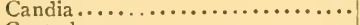 & 20 & 6 & 4 & 10 \\
\hline Canterbury..................... & $\mathbf{I}$ & I & . & $\mathrm{I}$ \\
\hline 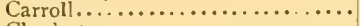 & 2 & .. & .. & - \\
\hline Charlestown................... & 6 & .. & 2 & 2 \\
\hline 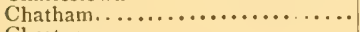 & 5 & .. & $\cdots$ & .. \\
\hline 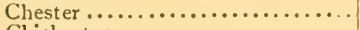 & 5 & . & .. & .. \\
\hline Chichester.................... & 7 & 2 & .. & 2 \\
\hline Center Harbor................... & 3 & .. & I & I \\
\hline Claremont....................... & 5 & $\cdots$ & I & I \\
\hline Cornish....................... & 20 & 3 & 3 & 6 \\
\hline Columbia....................... & 2 & .. & $\cdots$ & .. \\
\hline Croydon ....................... & 6 & . & .. & . \\
\hline Dalton...$\ldots \ldots \ldots \ldots \ldots \ldots \ldots \ldots$ & 5 & 2 & $\because$ & 2 \\
\hline Danbury $\ldots \ldots \ldots \ldots \ldots \ldots \ldots$ & 14 & $\cdots$ & 3 & 3 \\
\hline Deering...................... & 9 & . & . & . \\
\hline Derry......................... & 7 & $\because$ & 5 & 5 \\
\hline Dorchester...................... & 20 & 2 & 2 & 4 \\
\hline 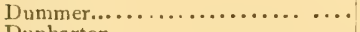 & 2 & *. & $\cdots$ & $\cdots$ \\
\hline Dunbarton..................... & 5 & . & -. & *. \\
\hline Durham ....................... & I & . & . & .. \\
\hline Easton $\ldots \ldots \ldots \ldots \ldots \ldots \ldots \ldots \ldots \ldots$ & 2 & .. & .. & .. \\
\hline Effinglam $\ldots \ldots \ldots \ldots \ldots \ldots \ldots \ldots$ & 22 & . & *. & -. \\
\hline Enfie!d........................ & 9 & $\cdots$ & . & - \\
\hline Epping....................... & II & .. & $\cdots$ & . \\
\hline 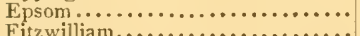 & 2 & $\cdots$ & $\ddot{0}$ & $\ddot{\theta}$ \\
\hline 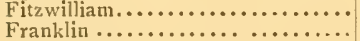 & 21 & $\cdots$ & 3 & 13 \\
\hline & 1 & $\because$ & * & $\because$ \\
\hline 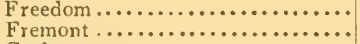 & 2 & $\mathbf{I}$ & I & 2 \\
\hline 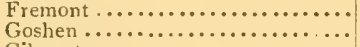 & 4 & 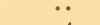 & 2 & 2 \\
\hline $\begin{array}{l}\text { Goshen } \ldots \ldots \ldots \\
\text { Gilmanton } \ldots \ldots \ldots \ldots \ldots \\
\end{array}$ & 12 & 4 & 2 & 6 \\
\hline 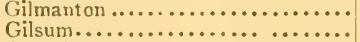 & 20 & I & 5 & 6 \\
\hline 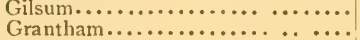 & 8 & 2 & 2 & 4 \\
\hline & 14 & $\mathbf{I}$ & . & I \\
\hline
\end{tabular}




\begin{tabular}{|c|c|c|c|c|}
\hline Towns. & 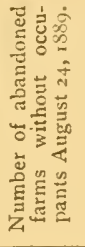 & 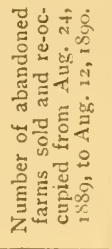 & 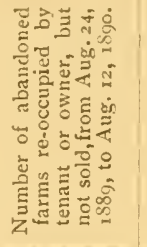 & 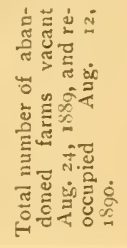 \\
\hline Greenfield.................. & 8 & 3 & $\cdots$ & 3 \\
\hline Greenland..$\ldots \ldots \ldots \ldots \ldots \ldots$ & I & $\cdots$ & . & $\because$ \\
\hline Greenville.................. & 2 & 1 & $\because$ & I \\
\hline Grafton $\ldots \ldots \ldots \ldots \ldots \ldots \ldots \ldots$ & 16 & 1 & I & 2 \\
\hline 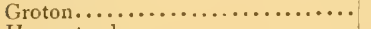 & 17 & $\cdots$ & 5 & 5 \\
\hline Hampstead $\ldots \ldots \ldots \ldots \ldots \ldots \ldots$. & 3 & I & $\cdots$ & I \\
\hline Hancock...................... & 5 & $\cdots$ & $\cdots$ & $\cdots$ \\
\hline 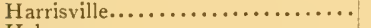 & I0 & $\cdots$ & $\cdots$ & $\cdots$ \\
\hline Hebron.$\ldots \ldots \ldots \ldots \ldots \ldots \ldots \ldots$ & 9 & I & 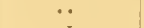 & 1 \\
\hline Henniker.................... & 7 & 2 & I & 3 \\
\hline Hill $\ldots \ldots \ldots \ldots \ldots \ldots \ldots \ldots \ldots$ & $\begin{array}{r}7 \\
27\end{array}$ & $\cdots$ & $\cdots$ & $\cdots$ \\
\hline Hillsborough $\ldots \ldots \ldots \ldots \ldots \ldots \ldots$ & 27 & $\because$ & ・ & 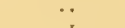 \\
\hline Hollis $\ldots \ldots \ldots \ldots \ldots$ & 3 & I & $\cdots$ & I \\
\hline Holderness.................. & 6 & $\cdots$ & 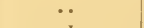 & $\cdots$ \\
\hline Hopkinton $\ldots . \quad \ldots \ldots \ldots \ldots \ldots$ & 10 & 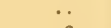 & $\mathbf{I}$ & 1 \\
\hline Hudson $\ldots \ldots \ldots \ldots \ldots \ldots \ldots \ldots$ & 12 & 2 & 4 & 6 \\
\hline Jaffrey $\ldots \ldots \ldots \ldots \ldots \ldots \ldots \ldots \ldots$ & 17 & $\cdots$ & I & $\mathbf{I}$ \\
\hline Kensington $\ldots \ldots \ldots \ldots \ldots \ldots \ldots \ldots$ & 2 & $\cdots$ & 2 & 2 \\
\hline Landaff $\ldots \ldots \ldots \ldots \ldots \ldots \ldots \ldots$ & 15 & 3 & 3 & 6 \\
\hline Lebanon $\ldots . . . \ldots \ldots \ldots \ldots \ldots$ & 1 & $\cdots$ & $\cdots$ & $\cdots$ \\
\hline Lee...$\ldots \ldots \ldots \ldots \ldots \ldots \ldots \ldots$ & 4 & $\cdots$ & $\cdots$ & $\cdots$ \\
\hline Lempster...................... & 14 & $\cdots$ & $\cdots$ & $\cdots$ \\
\hline Lisbon $\ldots \ldots \ldots \ldots \ldots \ldots \ldots \ldots \ldots$ & 3 & $\cdots$ & $\cdots$ & $\cdots$ \\
\hline Litchfield.... . . . . . . . . . . . & I & $\cdots$ & 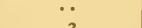 & $\because$ \\
\hline Littleton $\ldots \ldots \ldots \ldots \ldots \ldots \ldots \ldots$ & ${ }^{2} 5$ & I & 2 & 3 \\
\hline 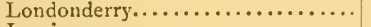 & 7 & 1 & $\cdots$ & I \\
\hline 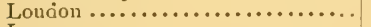 & 20 & 8 & $\cdots$ & 8 \\
\hline 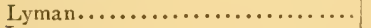 & 4 & $\cdots$ & 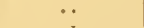 & $\cdots$ \\
\hline Lyme $\ldots \ldots \ldots \ldots \ldots \ldots \ldots \ldots \ldots$ & 3 & 1 & I & 2 \\
\hline Lyndeborough $\ldots \ldots \ldots \ldots \ldots \ldots \ldots$ & $\stackrel{4}{5}$ & 1 & $\cdots$ & I \\
\hline Madison $\ldots \ldots \ldots \ldots \ldots \ldots \ldots \ldots$ & 18 & 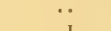 & $\cdots$ & $\cdots$ \\
\hline 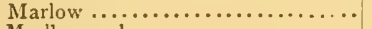 & 9 & 1 & $\cdots$ & I \\
\hline Marlborough ................... & I & $\cdots$ & 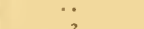 & $\cdots$ \\
\hline Meredith $\ldots \ldots \ldots \ldots \ldots \ldots \ldots \ldots$ & 5 & $\cdots$ & 3 & 3 \\
\hline Merrimack $\ldots \ldots \ldots \ldots \ldots \ldots \ldots \ldots$ & I I & $\cdots$ & 5 & 5 \\
\hline Middlet on ................... & 7 & $\cdots$ & 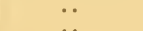 & $\cdots$ \\
\hline Milan $\ldots \ldots \ldots \ldots \ldots \ldots \ldots \ldots \ldots$ & 4 & $\cdots$ & $\cdots$ & $\cdots$ \\
\hline Milkon...$\ldots \ldots \ldots \ldots \ldots \ldots \ldots \ldots$ & 15 & 4 & $\because$ & 4 \\
\hline Mont Vernon................. & 5 & I & I & 2 \\
\hline Nelson.............................. & 7 & 3 & $\cdots$ & 3 \\
\hline Newport $\ldots \ldots \ldots \ldots \ldots \ldots \ldots \ldots$ & 13 & 1 & $\cdots$ & I \\
\hline 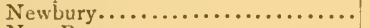 & 19 & $\cdots$ & $\cdots$ & $\cdots$ \\
\hline 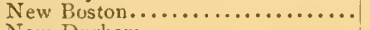 & 22 & 4 & 9 & 13 \\
\hline New Durham............... & 10 & $\because$ & $\cdots$ & $\because$ \\
\hline New London.................. & 9 & 1 & $\because$ & I \\
\hline Newmarket.'.............. & 5 & 2 & 2 & 4 \\
\hline Newington $\ldots . . . \ldots \ldots \ldots \ldots$ & 6 & $\ddot{*}$ & $\because$ & $\cdots$ \\
\hline 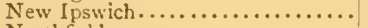 & 12 & 2 & 5 & 7 \\
\hline 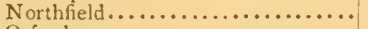 & 12 & 2 & I & 3 \\
\hline Orford $\ldots \ldots \ldots \ldots \ldots \ldots \ldots \ldots \ldots$ & Io & 2 & $\cdots$ & 2 \\
\hline Orange $\ldots \ldots \ldots \ldots \ldots \ldots \ldots \ldots$ & 1.4 & 2 & 5 & 7 \\
\hline Ossipee...$\ldots \ldots \ldots \ldots \ldots \ldots \ldots \ldots$ & 4 & $\cdots$ & $\because$ & $\because$ \\
\hline 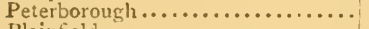 & 7 & 2 & 2 & 4 \\
\hline Plainfield..................... & 13 & 2 & 3 & 5 \\
\hline 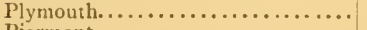 & 2 & 2 & $\cdots$ & 2 \\
\hline Piermont.................... & 9 & $\because$ & $\cdots$ & $\because$ \\
\hline Pittsfield $\ldots \ldots \ldots \ldots \ldots \ldots \ldots \ldots \ldots$ & 3 & I & $\because$ & t \\
\hline 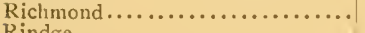 & 19 & I & 3 & 4 \\
\hline Rindge...................... & II & 2 & 3 & 5 \\
\hline
\end{tabular}

\section{Lor}




\begin{tabular}{|c|c|c|c|c|}
\hline Towns. & 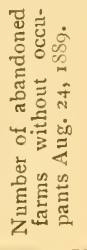 & 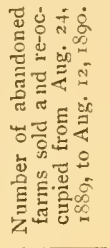 & 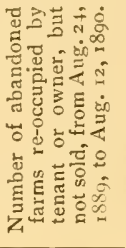 & 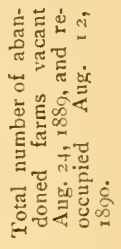 \\
\hline Rochester................... & 5 & 2 & $\cdots$ & 2 \\
\hline Raymond $\ldots \ldots \ldots \ldots \ldots \ldots \ldots$ & 14 & 2 & 2 & 4 \\
\hline Roxbury $\quad \ldots \ldots \ldots \ldots \ldots \ldots$ & 6 & $\cdots$ & $\cdots$ & $\cdots$ \\
\hline Salisbury... $\quad \ldots \ldots \ldots \ldots \ldots \ldots$ & 9 & $\cdots$ & $\because$ & $\cdots$ \\
\hline 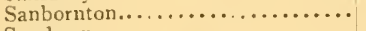 & 12 & $\cdots$ & 2 & 2 \\
\hline Sandown $\ldots \ldots \ldots \ldots \ldots \ldots \ldots$ & 3 & 2 & $\cdots$ & 2 \\
\hline 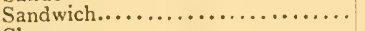 & 20 & 2 & $\cdots$ & 2 \\
\hline 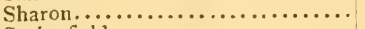 & 5 & 2 & $\cdots$ & 2 \\
\hline Springfield $\ldots \ldots \ldots \ldots \ldots \ldots \ldots$ & I I & 4 & . & 4 \\
\hline 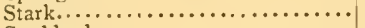 & I & $\cdots$ & $\cdots$ & $\cdots$ \\
\hline Stoddard ...... $\quad \ldots \ldots \ldots \ldots$ & 14 & $\cdots$ & $\cdots$ & .. \\
\hline 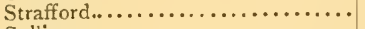 & 5 & $\cdots$ & $\cdots$ & $\cdots$ \\
\hline 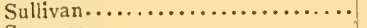 & 7 & I & $\cdots$ & $\mathbf{I}$ \\
\hline Sunapee.................... & 6 & 2 & I & 3 \\
\hline 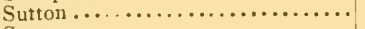 & 13 & $\cdots$ & 5 & 5 \\
\hline Swanzey...$\ldots \ldots \ldots \ldots \ldots \ldots$ & 18 & I & 6 & 7 \\
\hline 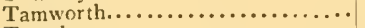 & 3 & $\cdots$ & $\cdots$ & $\cdots$ \\
\hline Temple .................... & 8 & I & $\cdots$ & I \\
\hline 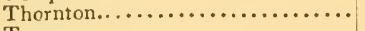 & I4 & 4 & 1 & 5 \\
\hline Troy...$\ldots \ldots \ldots \ldots \ldots \ldots \ldots$ & 8 & $\cdots$ & 2 & $\cdots$ \\
\hline Tuftonborough $\ldots \ldots \ldots \ldots \ldots \ldots$ & 4 & $\cdots$ & $\cdots$ & $\cdot$ \\
\hline Unity,$\ldots \ldots \ldots \ldots \ldots \ldots \ldots \ldots \ldots$ & I 1 & $\cdots$ & $\cdots$ & $\cdots$ \\
\hline 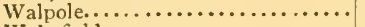 & II & $\cdots$ & $\cdots$ & $\cdots$ \\
\hline Wakefield.................. & 12 & 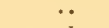 & $\cdots$ & $\cdots$ \\
\hline Warner $\ldots \ldots \ldots \ldots \ldots \ldots \ldots \ldots$ & 5 & $\mathbf{I}$ & $\cdots$ & I \\
\hline Warren...$\ldots \ldots \ldots \ldots \ldots \ldots \ldots$ & 2 & 2 & $\cdots$ & 2 \\
\hline Washington.$\ldots \ldots \ldots \ldots \ldots \ldots \ldots$ & 6 & 1 & . & I \\
\hline IVeare...$\ldots \ldots \ldots \ldots \ldots \ldots \ldots \ldots \ldots \ldots$ & 17 & 1 & 4 & 5 \\
\hline Webster...................... & 5 & $\mathbf{I}$ & $\cdots$ & $\mathbf{I}$ \\
\hline Wentworth $\ldots \ldots \ldots \ldots \ldots \ldots \ldots \ldots$ & 7 & $\cdots$ & $\cdots$ & $\cdots$ \\
\hline Westmoreland $\ldots \ldots \ldots \ldots \ldots \ldots \ldots$ & I I & I & 3 & 4 \\
\hline Wilmot $\ldots \ldots \ldots \ldots \ldots \ldots \ldots \ldots \ldots$ & II & $\because$ & 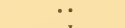 & $\cdots$ \\
\hline Wilton $\ldots \ldots \ldots \ldots \ldots \ldots \ldots \ldots \ldots$ & 7 & 2 & $\mathbf{I}$ & 3 \\
\hline Windham $\ldots \ldots \ldots \ldots \ldots \ldots \ldots$ & 7 & $\cdots$ & 2 & 2 \\
\hline 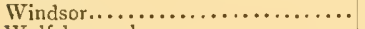 & 4 & $\cdots$ & $\cdots$ & $\cdots$ \\
\hline Wolfeborough $\ldots \ldots \ldots \ldots \ldots \ldots$ & II & 2 & $\cdots$ & 2 \\
\hline Totals.................. & 1,342 & $1+1$ & 160 & 301 \\
\hline
\end{tabular}


Gratifying as these facts may be, they are doubly increased in significance when we consider that the class of people purchasing and renting these farms is generally such as appreciates the grand scenery, healthy climate, and association with an intelligent class of people, for all of which New Hampshire has become noted. Those farms which have become occupied for agricultural purposes are mainly taken by Americans, and we believe they will have the energy and ability to succeed. A large number of these farms have become occupied by city people to whom a home in the country has been a fond anticipation for many years, and it is needless to say that they will find in a New Hampshire farm all the opportunities for gratifying any worthy desire in establishing a home for comfort, health, or pleasure. They come, not to make money but to spend it, and fortunate indeed will it be for the State when the unsold abandoned farms are utilized by this class of people. We are informed from creditable sources that the summer-boarding business was never so large in the State as it is the present season, and it is not unreasonable to suppose that the wide-spread attention recently called to the attractions of New Hampshire has had some effect in this direction. We believe that the appreciation, both at home and abroad, of New Hampshire's advantages which has surely resulted from the enactment of this law, can be strengthened and extended by a liberal policy on the part of the State until the capacity of our summer hotels and boarding-houses will need to be doubled, and no abandoned farm worth any one's attention need be without occupants.

Respectfully submitted.

N. J. BACHELDER,

Commissioner. 



LIBRARY OF CONGRESS
0029407484

LIBRARY OF CONGRESS
00029407484

LIBRRRY OF COACERESS

LIBRARY OF CONGRESS
0 002940748 .

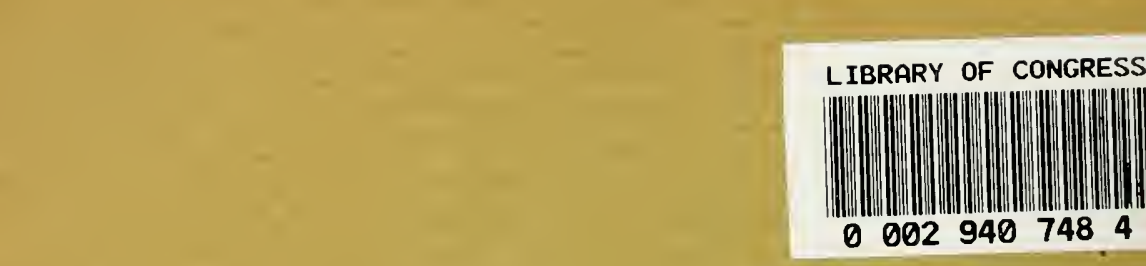

